longer, he said it might bring her in danger of a violution of the protectors proclamation or - and that wood shorten his life and in the menetime fill it with fere and so make it uncomfortable.

I hsve told

LETTER FROM GEORGE HICERS, D.D., DEAK OF WORCESTER.

TrE following letter from Dr. George Hickes, dean of Worcester, eminent alike as scholar and nomjuror, will be read with much interest. It relates to his brother, John Hickes, the nonconformist minister on whose account Alice Lisle suffered so cruelly and who was himself executed at Glastonbary on 6 Oct. 1685 for sharing in Monmonth's rebellion. Of him we are told by Calamy that he was ejected in 1662 from Stoke Damerel in Devonshire, and then settled at Portsmouth. His dying speech, together with three letters to his wife and one to a nephew, is printed in the 'New Martyrology ; or, the Bloody Assizes,' written by John Tutchin but published anonymonaly ; of which, however, the fourth edition, issued in 1699, bears the name of Thomas Pitts as anthor. The speech is also given in Will. Turner's ' History of the most remarkable Providences,' published in 1697. It furnishes some interesting particulars of his life; that he was educated st Dublin; was acquainted with colonel Blood after 1671, bat was not engaged in any of his plots, although Blood procured a pardon for him from the hing when 'involved in great trouble of another nature,' of which he says he had given a narrative to the world; that when the duke of Monmonth landed, he went directly to him at Shepton Mallet from the east conntry ; that he was betrayed by one Barter of Lisnel [?], who was ' such a traitor' to the dake, 'his old and intionste friend ;' that he joined the duke, believing in his legitimacy and his title to the orown; and that he died 'owning my ministry, nonconformity, for which I have suffered so much, and which doth now obstruct the king's grace and mercy to be manifested and extended to me,' but disclaiming all rebellious principles. On torning to the Dublin 'Catalogue of Graduates' (printed in 1809), I find that John Hickes took the degree of B.A. 4 May 1655.

With all allowance for strong differences in religions and political views, and probable consequent separation in life, the tone of the dean's letter seems somewhat hard; and one is glad, therefore, to learn from John Hickes' letters that his brother made some attempt to save him. In his letter to his nephew on 5 Oct. he says: 'I wrote last Batorday was a sevennight to my brother Gearge, but whether he is at London or Worcester, I know not; I wrote to him to desire him to petition the king, that some farour and merey might be shewred me, if he thought fit;' and then in one of the letters to his wife he says, 'Monday lest my brother' (no doubt his brother Gearge) 'went to Irondon to try what could be done for me; what the success will be, I know not.'

He was, however, as nnfielding in his own principles as was the dean ; he says to his wife, 'I bless God who hath kept me from all temptations to conformity,' and asserts that it is his courage and public spirit for the protestant religion and the English liberties that have brought him to this end. He mentions his two children, James and Betty. 
A singularly hard fate attended the consistency of the two brothers: the one ejectea for nonconformity to the church. and executed for rebellion against the ling; the other, ejected for anvielaing conformity to what he conceived to be the true church principles of obedience. and suffering loss of all preferments and worldily wesith for stannch loyalty to the very same hing bat five vears afterwarts.

The notice of Bishop Kon's pious charity will not escape readers.

The original of the letter is now in the Bodleian Library, having ben given in the present year by Mrs. Arthur Erans. at whose disposal it was placed by the owner, Mr. Frederick Lrocker-Lampson. It conld not be in a more suitable place of deposit. since the Library possessed already many of the dean's letters and papers.

Writuar Duns Mackay.

Much hond $\mathrm{Sr}$

Worcester Oct $17^{\text {th }} 85$.

At my retarn to the Deanery from visiting the manors belonging to onr church 2 dayes ago. I found yone very kind letter, for wch I retarn you my most hearty thanks arua will ever ackoowlege your great charity, and respects towrids my late wretched brother, wet shail remsin a debt upon my account. as long as I live.

I must also entreat you to return my most humble duty and thankes to my good Lord Bishop for his eminent condescenscion, and charity towards him in praying w $^{\text {th }}$ bim, and for him. and for soffering so unworthy a body to be interred in Glsssenbary-Charch.

I take this last great respect of my Iords to be don to myself. and desire in a particular manner to be thankfull for it. I am glad be mado such professions of his logalty, and gave the people such good exhortations to be true, and faithfull to their lawfull soveraign, and to detest all manner of rebellion, bat am verr sorry $\mathrm{g}^{t}$ he persisted in justifying his nonconformity : this part of his last behavionr filles my heart $w^{\text {th }}$ greif, tho' I was prepared to expect it, as bnowing very well how ignorant he was of the true natare of charch-communion, and how much he was prepossessed $w^{\text {th }}$ folse notions and principles in mstters relating to churchdiscipline and government.

I humbly intreat you to send on the paper he delivered to you, yon may direct it to me at the Desnery in Worcester, and I also pray you to let me know, whether he left any charge, or message to his children in word or writeing, $\mathrm{y}^{\mathrm{t}}$ they shoold live in the communion of our church and whether he desired, and received the holy sacram", and if not, whether he refused it, or it was refused to him, as might justly have been don to a man persisting in schisme. I also desire to know, whether his body was delivered whole to his friends, and if so, whether it was don by order from my Lord Ch. Justice : I wrote to his Lordp to beg so much mercy of him, and if he granted my petition, it is fit I should know it, and give him thenkes.

I should be also glad to boow what my Lord said to him at his tryall, and condemnation. and whether he said anything to the people in justifi. cation of his nonconformity at the time of his erecution, and if he acbrowleged bis punishment to be the righteous judgm tof God for his sin of rebellion. 
There is a worthy gentleman of the charch of Welles, to whome I beseech you give my hamble service, and particular respects, I mean $D^{r}$ Creighton, and to the good Dean, if he be there.

I doubt my curiosity hath made me too troublesome to you, but I assure you, yon may in requiteall command me any service, for I am in all sincerity

\section{dear $\mathrm{Br}^{\mathrm{r}}$}

Your most obliged, affect. and humble serv ${ }^{t}$

GEORGE HickE8.

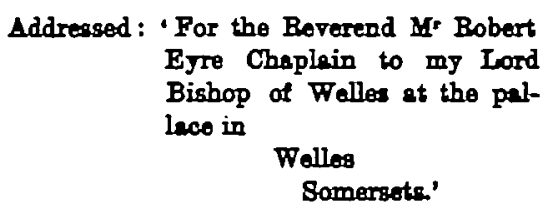

Postmark, ' in all 6d.'

LORD MCACAULAY AND THE ABSAULT OF NAMOR.

Consmerang the repatation as a historian in which Lord Macsulay is beld, it is well to point out any important insccuracies that may have occurred in his picturesque descriptions. The subject to which the present remarks refer is his account of a prominent historical event, viz. the assault of Namur citadel in 1695.

It was during the war in which Grest Britain, Holland, Spain, and the Empire were in conflict with France, that, in July 1695, the strong fortress of Namur in the Netherlands, then in the hands of Louis XIV, was invested and besieged by the allies under the personal command of William III. After an attack of nearly two months' duration, when the Duc de Boufflers had effected a skilful and vigorous defence, but Villeroy, who with a French army had intended to relieve him, was obliged to retreat, the crowning act of the siege wre resolred upon. This was the general assault of the covered way (or counterscarp, as the expression then ran) of the citadel, and of the breaches which had been made in the Terra Nove and Coehorn outworks. Of the share taken by the English troops in this hard-fought operstion the following is the account given by Irord Macarlay:-[1] 'The troth is that most of the [English] regiments which had seen service had marched with William to enconnter Villeroy. [2] Cutts at the head of a small body of grenadiers marched first out of the trenches with drums beating and colours flying. [8] This gallant band was to be supported by four battalions which had never been in sction, and which, though foll of spirit, wanted the steadiness which so terrible a service required. . . [4] The ram recruits [on Catts being wounded], left almost without direction, rushed forward impetuonsly till they found themselves in disorder and out of breath. . . Ther lost heart and rolled back in confusion till Cutts . . . sacceeded in rallying them' (Macaulay's ' History of England,' iv. 594).

Among the contemporary records of this event, Auvergne's ela- 
borate history is clearly the standard. It is the only work treating solely of the war in question; it was published in successive volumes during the progress of that war (thus the events of 1695 were given to the world in 1696); whilst on its descriptions of the campaigns of William III the accounts given by every English history of the eighteenth century have been founded, and the official records of the British army have been based. But in addition to Auvergne's work there is the following important confirmatory testimony:-the London Gasette of 29 Angust, 1695; the 'Exact Journal of the Siege of Namar,' published in 1695; the 'Campaigns of King William and the Duke of Marlborough,' by Brigadier-General Richard Kane, who actually took part in the assandt of Namur, though his work was not published till after his death in 1745; and Bishop Kennet's 'Complete History of England to the Desth of his Majesty King William III,' pablished in 1706. There is also Boyer's 'History of William III,' published in 1708, but owing to there being no copy of this work in the British Museam the present writer has been unsble to peruse it. Even if it differ from Auvergne it cannot compare with that history in point of authority. But the evidence furnished by later writers of the eighteenth century-i.e. of Ralph, Smollett, and Tindal, who doubtless had soen Boyer's account-goes to show that nothing in it is antagonistic to the statements of Auvergne, Kane, and Kennet.

Having tested Lord Macaulay's version by the light of these records, we will reply to it in detail, taking separately each numbered group of assertions.

(1) When the assault was delivered, 'most of the regiments which had seen service ' had not marched with William to encounter Villeroy, for the force which had been actually detached from the besneging army under William's personal command, to strengthen the covering army of the Prince of Vaudemont in opposing Villeroy, only included the following nine English bettalions, viz.:- - five battalions of the brigade of Guards, Columbine's regiment (6th), Stanley's (16th), Seymour's (24th), and Lander's; the majority of the regiments which had seen service, numbering thirteen battalions, having been left behind. But even the nine detached battalions had only proceeded as far as Masy-a distance of no more than six miles-where lay the prince's camp. Moreover, each of these nine battalions furnished a body of grenediers to talke part in the assault, and William himself wes actually present thereat. It was owing to Villeroy's retreat from before Masy that the hing ordered the assandt to be made; and, so far as can be seen, its unsuccessful result was in nowise brought about, or even conduced to, by this detachment of some of the regiments to Masy.

(2) The 'small body' of grenadiers, as Lord Macaulay styles them, numbered at least 700 men, and constituted the troops who practically delivered the assault. In eddition to the nine battalions already mentioued, the following nine also supplied grenadiers, viz.:-the Royals, Selwyn's (2nd), Trelawny's (4th), Royal Fusiliers (7th), Tidcomb's (14th), Ingoldsby's (23rd), Maitland's (25th), Collingwood's and Saunderson's. In fact, the bront of the assanlt was effected by these picked troops of the English army. But they were not headed or accompanied by drums 
and colours which belongea to the complete regiment forming the supports.

(8) The supports consisted solely of one battalion-Courthope's (17th)-numbering about $500 \mathrm{men}$. It had been intended that Mackay's (Scots) regiment of sbout equal strength should hsive sccompanied Conrthope's. but on account of the crowded state of the adranced trenches, Macksy's had to be drawn up with the reserves at Salsines Abbey, some distance in rear, and half a mile from the breach; and accordingly it acted with them. Altogether the reserves (whom Lord Macanlsy confuses with the supports) numbered three battalions. or about $1.500 \mathrm{men}$, the other two regiments being Frederick Hamilton's Irish (18th)-in which Bane served-and Bnchan's Seots. As regards the assertion that these four regiments, constituting the supports and reserves, 'bad never been in action,' it is to be noted that, though apparently Courthope's and Hamilton's had not yet shared in any great battle or siege operation, yet they hed but recently taken part in Vaudemont's arduous campaign against Villeroy, and especially in the prince's calebrated retreat in the face of the enemy from Aarseele to Marykirk. Bnt Mackay's had actually fought at the grest battles of Steinkirk (1692) and Landen (1698), whilst in June of this very year 1695 Buchan's had fought onder the duke of Wütemberg in the bloody and unsuccessful assault made on Fort Knooque. Tte alleged 'want of stesdiness' of these four regiments will be considered under our next head.

(4) It will be noticed thet Lord Macsulsy, having dismissed his 'small body of grenadiers,' now fathers the main action of the assent on the raw recruits,' into whom (apparently on account of the supposition that they 'had never been in action ') be has converted his four supporting battalions. These troops, we are told, " wanted the stesdiness which so terrible a service required,' ' found themselves in disorder,' ' lost heart ' and 'rolled back in confasion.' Let us now look at the facts. The sassait was delivered by a strong force of grenadiars, supported by Courthope's regiment of cansiderably less strength. Amidst a desdly fire both in front and in flank, these devoted men advanced to the storm most resolutely and steadily, and, so far as can be accurately gathered, mast of them were placed hors de combat before they even reached the foot of the breach. The loss in officers especislly was immense. Through a mistake.in the signals the three reserve battalions at Salsines Abbey were not ordered to advance sufficiently soon after the main body to render efficient assistance. They marched fortrard equally bravely and in like good order; but on sccount of the loss of time in starting, and the distance they had to pass over, their advance constituted a distinct second sasarlt, which was equally unsuccessful with the first, though Hamilton's Irishmen managed to gain the summit of the breach before they were repulsed. In neither of the attacks did the soldiers evince the slightest ' want of stendiness,' nor was there any 'disorder' apart from what is inseparable from the effects of a murderous fire closely directed on advanoing colomns of troops; not a single officer or soldier 'lost heart;' nor was there any 'rolling back in confusion,' nnless this be the proper literary phrase by which to denote the retrest of the remnants of a brave storming party. It was woder the eyes of the 
hing (whom Lord Macaniay states to have been at the time confronting Villeroy) that this assauit took place; ana so pleasea was he at the excellent conduct of Hamilton's men thet he gave them the title of 'the Rojal Regiment of Foot of Ireland ' (afterwards changed to the Royal Irish Regiment), his own arms, the lion of Nassau, with the harp and crown, and the motio Dirtutis Namurcencis Promium, all of which distinctions they bear to this day. [Anvergne (1296), 141-155; Kano, 23-25; Eennet, iii. 697; Lonaion Gaserte, 29 Aug. 1695; 'Exact Journal,' 26; Raiph, ii. 604; Smollett, i. 257; Tindal, xiv. 237; Cannon's 'Offeisi Becords,' 17th and 18th regimenis; Hamilton's 'Grenadier Guards,' i. 400-402.]

ARTHUR PARMrLC.

\section{SPANHEDY'S ACCOUNT OF THE RNGLISH COURT.}

Ezkchine Spantrens was born at Geneva, December 7, 1629, and died in London, November 14, 1710. In the service of the Elector-Palstine, Charles I-epris, and aiterwards for many years as amisassador of the Grear Elector of Brandenburg and of Frederick, the first hing of Prussia, he displayed a vigorous diplomatic acivivity in the scenes of high European policy. A valusble witness to the keenness of his observation and the impartiality of his description exists in the 'Relstion de la Cour de France' of the year 1690, which has recently been carefully edirea by M. C. Schefer.' But it has not bitherio been known thas the ambassador also drew up a 'Belation' on the English courr. Ranke himself makes no mention of it, though he has used Spanheim's accounts. No doubt this 'Portrait,' both in dimensions and in content, is inferior to the 'Belation de la Cour de France.' Still in its characterisation of queen Anne, in its statement of the relations of the princess towards queen Mary and her consort, in its picture of the duire and duchess of Marlborough, we see throughout an endesvour to do justice to the persons

- Relation de la Cour de France en 1690, par Extehial Spanheire Pur M. C. Sahefer (Paris, 1882). The form of the tart given in this edition cannot, however, be regarded as definitive (of Bocher, in Sybel's Historiscine Zoitecinoifh, Iv. 316). M. Sahoter hed, anformanstely, no bnowledge of a second menuseript in the Geheimes Stalsarchiv; and an exect collation of the four existing redicotions of the wort might

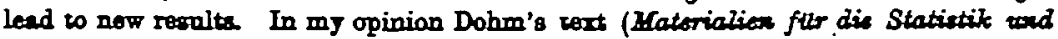
nowere Stactonkusdo, iii 168-286, จ. 1-218; Lemgo, 1780, 1785) is besed upon * draft which does not contain Spanheim's corrections and additions. Of the two manubcripts in the Geheimes Stretsarchiv at Berlin, one (R. 94) presenter, besides the original composition which is tor the must part atill recognissbie, nomeroms anrograph improvements and additions by Spanheim; whiie the other (R. xI: of Schefer, Len, intr. pp. zurv. rnviii) is a tair copg with less nomerous notes of Spanbeim's, and hes tho editions, to which wo hevo referred already, incorporeted in the tert. Both oopies contain the Convidtrations sur la situation presents (Sahefer, Pp. 346-388), which are not in Dohm. The msnuscript in M. Schefer's possession comseins on the one hand-corresponding to the first Berlin copy-cacograph additions if Spanheim, while it lacks certain pieces (indicated by Scheter, Pp. 432-414), which are to be found at least in part in the three other redections, and are, moreover, mostly distinguished by bracizets in the first Berlin manuseript. Consequently it is likely that M. Schefer'B copy is a trenecript abridged sceording to Spanheim's choice, and furmished by him with adaitions. 
and circumstances portrayed, together with a temperate judgment, resulting from the experience of a long life. It is a relation, too, in which the author is guided by a conscientions desire for accuracy and sense of truth."

The 'portrait' is preserved in the Geheimes Staatsarchiv at Berlin. and consists of thirteen lesves in folio. It may be questioned whether this, the only manuscript known to exist, contains the work in its entirety, In one place (p. 764), Spanheim mentions a section dealing with the queen's relations with foreign ambassadors, which is nowhere to be found. The account, too, ends without any appropriate conclusion. And yet the character of the manuscript-a fair copy with antograph additions and corrections by the ambassador-does not permit the assumption that it is a fragment.

As for the origin of the 'Relation' $7 e$ have more exact information than in regard to that of the year 1690. On August 7, 1704, an order of king Frederick I was addressed to Spanheim with these instructions :-AL diewoil Wir auch von dor jotrigen Königin von England Persohn, naturel und qualitaten gern genaus Nachricht haben mögton und Jhr vor Zoiten von dem Bönige in Frankreich und dohnen vornehmsten Persohnon seines Hauses und Hoffes dorgloichen woll oingerichtete Portraite gemacht und ubergeben habet, so wollt Thr auch eins von gedachtor Königin zu Papir bringen. On the $\frac{1}{2}$ Angast Spanheim reports: Comme d'un cote, Sire, jo mo trouve bion glorieux du temoignage avantageuse qu'il luy plaist de rendre aux Portraits surmontionnes du Roy de France et des Personnes de sa Maison, jo ne pourrai de l'autre que m'acquitter avec autant de zelo, de promtitude et de confiance de celuy, qu'Elle desire que jo fasse de cette Reine. C'est aussi d quoy jo ne manquerai pas de travailler incessemment. On October 10 follows the ling's ackmowledgment: Das portrait so Jhr von I. M. gemacht und Uns eingesandt, hat dis estime so Wir vor diselbe jeder Zeit gehabt, nicht wenig bei Ons vermehrt und finden Wir solbiges sehr woll eingorichtet.

After the example of $M$. Schefer we give the text word for word, retaining all peculiarities of language as well 83 the punctuation corrected by Spanheim himself.

R. DoRBNBR.

Barlin.

\section{Portrait de la Reine d'Angleterre.}

Vostre Majesté m'ajant ordonné de lay faire le Portrait de la Reine d'Angleterre aujourd'huy regnante, le plus naif et le plus ressemblant, qu'il se pourroit, je ne puis que taschér a m'en aquitter, avec toute l'attention et ls soumission requise. Ce qui ne pourrs que donner lien à ls representer dans la constitation de sa personne; dans ses qualités et ses inclinations, et ainsi ${ }^{3}$ le veritable caractere de son esprit; dans sa conduite domestique, on \& l'égard da gonvernement et des affaires; dans ls consideration de ses Ministres, à qui Elle s'en rapporte le plus, ${ }^{4}$ et qui ont le plus de part a sa confidence et a son estime; ou des Dames, en qui Elle a le plus de créance; enfin dans ce qui pent regarder l'estat present de sa Cour, et particulierement par rapport aux Ministres étrangers, qui y sont envoyés. I y a quelques autres circonstances à l'égard de sa

2 Compare the prefece to the Relation of 1690 , Schefor, La, intr. p. xrr.

- Airui added by Spanheim.

- Spanboim has corrected le plus instead of davantage. 
and circumstances portrayed, together with a temperate judgment, resulting from the experience of a long life. It is a relation, too, in which the author is guided by a conscientions desire for accuracy and sense of truth."

The 'portrait' is preserved in the Geheimes Staatsarchiv at Berlin. and consists of thirteen lesves in folio. It may be questioned whether this, the only manuscript known to exist, contains the work in its entirety, In one place (p. 764), Spanheim mentions a section dealing with the queen's relations with foreign ambassadors, which is nowhere to be found. The account, too, ends without any appropriate conclusion. And yet the character of the manuscript-a fair copy with antograph additions and corrections by the ambassador-does not permit the assumption that it is a fragment.

As for the origin of the 'Relation' $7 e$ have more exact information than in regard to that of the year 1690. On August 7, 1704, an order of king Frederick I was addressed to Spanheim with these instructions :-AL diewoil Wir auch von dor jotrigen Königin von England Persohn, naturel und qualitaten gern genaus Nachricht haben mögton und Jhr vor Zoiten von dem Bönige in Frankreich und dohnen vornehmsten Persohnon seines Hauses und Hoffes dorgloichen woll oingerichtete Portraite gemacht und ubergeben habet, so wollt Thr auch eins von gedachtor Königin zu Papir bringen. On the $\frac{1}{2}$ Angast Spanheim reports: Comme d'un cote, Sire, jo mo trouve bion glorieux du temoignage avantageuse qu'il luy plaist de rendre aux Portraits surmontionnes du Roy de France et des Personnes de sa Maison, jo ne pourrai de l'autre que m'acquitter avec autant de zelo, de promtitude et de confiance de celuy, qu'Elle desire que jo fasse de cette Reine. C'est aussi d quoy jo ne manquerai pas de travailler incessemment. On October 10 follows the ling's ackmowledgment: Das portrait so Jhr von I. M. gemacht und Uns eingesandt, hat dis estime so Wir vor diselbe jeder Zeit gehabt, nicht wenig bei Ons vermehrt und finden Wir solbiges sehr woll eingorichtet.

After the example of $M$. Schefer we give the text word for word, retaining all peculiarities of language as well 83 the punctuation corrected by Spanheim himself.

R. DoRBNBR.

Barlin.

\section{Portrait de la Reine d'Angleterre.}

Vostre Majesté m'ajant ordonné de lay faire le Portrait de la Reine d'Angleterre aujourd'huy regnante, le plus naif et le plus ressemblant, qu'il se pourroit, je ne puis que taschér a m'en aquitter, avec toute l'attention et ls soumission requise. Ce qui ne pourrs que donner lien à ls representer dans la constitation de sa personne; dans ses qualités et ses inclinations, et ainsi ${ }^{3}$ le veritable caractere de son esprit; dans sa conduite domestique, on \& l'égard da gonvernement et des affaires; dans ls consideration de ses Ministres, à qui Elle s'en rapporte le plus, ${ }^{4}$ et qui ont le plus de part a sa confidence et a son estime; ou des Dames, en qui Elle a le plus de créance; enfin dans ce qui pent regarder l'estat present de sa Cour, et particulierement par rapport aux Ministres étrangers, qui y sont envoyés. I y a quelques autres circonstances à l'égard de sa

2 Compare the prefece to the Relation of 1690 , Schefor, La, intr. p. xrr.

- Airui added by Spanheim.

- Spanboim has corrected le plus instead of davantage. 
naissance, de son âge, et de son estat précédent en qualité de Princesse de Dennemarc, qu'il sera à propos de toucher icy par avance et sommairement.

De la Boine d'Angleterre, avant son avenement at ta Couronne, de sa naissance, et de la Duchesse d' Yorc sa Mere.

La Reine d'Angleterre aujourdhny regnante, est née le 26 Ferrier en 1666, suivant le Calendrier d'Angleterre, qui ne commence l'année qu'au 25 de Mars, on en 1667, suivant le Calendrier de dela la mer. Elle est fille, comme on scait, du fen Roy Jacques II, alors Duc d'Yorck, appellé la Princesse Anne, et scear cadette de son aisnée, lo Princesse Marie, en suite Princesse d'Orange, et depais Reine d'Angleterre aveo le fen Roy Guillsame son Epoux. Lcu- Mere étoit fen lo Duchesse d'Yorck, Anne Hyde, premiere femme de ce Iuc, fille du Chancelier d'Angleterre Clarendon que ce mesme Duc frere ciu Roy Charles $I$ aroit épousée secretement, durant leur retraite dans les pays étrangers, dn vivant de Cromvel. II declara son mariage apres le retablissement da dit Roy son frere sur le Throne d'Angleterre, en l'année 1660. Quant an Chancelier d'Angleterre, Comte Clarendon, Pere de la Duchesse, il étoit né d'une condition de simple Gentilhomme, appellé Henry Heyden, Docteur aux Loix de sa profession, et qui sous le regne de Charles I s'estoit attaché an parti de la Conr, dans le long Parlement qu'il g eut, et en soutint les interêts dans lo Chambre des Commanes, dont il estoit membre. Comme il suivit Charles III, alors Prince de Gales dans sa retraitte, hors d'Angleterre, il y demeura attaché à sa personne, et à son service, et comme son principal Conseiller, en fat déclaré Chancelier. Apres le retablissement da dit Charles II sur le Thrône, il fut conservé ou établi dans la dite charge de Chancelier d'Angleterre; cré Mylord Baron en la mesme année 1660 et Comte l'année suivante 1661. Lo mariage de sa fille avec le Due de Yorck, le presomptif héritier de la couronne [reu le peu d'apparence que le Roy Charles II vint a avoir des enfans de la Beine son épouse, et encore virante on Portugall et ainsi a pouroir devenir Reine d'Angleterre, ne manqua pas d'attirér l'envie et le blâme contre le dit Chancelier. A quoy servit de preterte on de fondement, le mariage da Roy avec une Princesse Cátholique Romaine et sterile, comme estant ${ }^{5}$ procuré a dessein d'en favoriser l'elévstion de sa fille à ls qualité susdito de Roine; et ensoite la rente de Danquerque à lo France, pour quatre millions de livres de France, et qu'on attribuoit aur conseils da dit Chancelier, comme alors le promier et plas accredité Ministre dn Roy Charles II. Le dit Roy g'etant dégousté dans la suite de ce Ministre; et entr'antres ajant sçon qu'il anroit sous main porté une Dame, ${ }^{6}$ qu le Roy aimoit, à preferer le parti d'epousér secretement le Duc de. Richemont, un des premiers Seigrears du Royanme, \& celuy d'estre sa Maistresse, il abandonna le dit Chancelier a la haine, ou envio de ses ennemis. Ce qui donno lien a ce Ministre de chercher sa seureté hors du Royanme, et is se retirer en France, où il est mort. Il laisse deux fils en Angleterre, tous deux encore en vie;

- Estart, added by Spanheim.

- Merginal note by Spanheim : C'estoit une Demoisclle nommis Marie Sheart at qui ost morts seulement (f) icy d Londres dopuis pou de moy en ga. 
l'um qui porte son tiure de Comre de Clarendon; et l'antre, celuy de Comte àe Rocinester, à quoy il a esté elevé par ie feu Roy Cinsrles, en $160^{\circ} 2$.

Pour is Duchesse d'Yorck Mere de lo fen Raine Marie, er de ls Reine Anne regnante, eile mourut sans avoir laisse dautres enrans ni winsi ancun tils masle, Elle se deciars Caibolique Romaine ar liet dè se mort, y ayunt esié portée, comme on peut croire, par le Duo son Ipoux; ei qui depuis qu'ii s'est retiré en France, et peu de tems spant sa mort, y İt imprimer les circanstances de certe áéclarakion de fen la dite Duchesso son Epouse, de mesme que ${ }^{i}$ celle du feu Roy Charies II son ìrere, iaice pareiliement avant su mort.

\section{De l'eaucation de cetue Princesse et de son mariage avec lo Prince de Tannemarc.}

Is Princesse Anne iủt elevée avec la Princesse Marie son aisnée an Cokpir, qui est consiäaré comme une partie de Whitehsll, regarīant sur le Parc de S. Jemes, et dans le mesme appartement, qui m'y fứt assigné à mon défrayement, ensuite ảe mon entré publique à Ionäres, en qualité d'Ambrssadeur Extrandinsire de Vv Majesté. ${ }^{\circ}$ L'éducation de ces dear Princesses se fit avec peu de pompe, et sous la conduite de leurs Gonvernuntes. J'y eus l'honneur d'en avoir andiance, en mon premier envoy en ángieterre en $1675^{\circ}$ de la part da fen Electear Palatin Charles Louys. In Princesse Marie estant mariée l'anné 1677, ${ }^{10}$ avec le Prince d'Orange son consin germain, et depuis Roy d'Angleterre Guillanme III, la Princesse Anne rests an dit Cö́pit jusques en $1683,{ }^{11}$ qu'Elle fust mariée avec le Prince George de Dennemarc. Ce mariage int mis sur le tapis ct negorié par ia France, qui étoit alors dans one granäe liaison aveo le Dennemare, de meme 12 qu'aveo le Duc d'York. Comme j'estois alors Envoyé de la part de fou Sa Serenissimeté Electorale de gloriense memoire en la aite Cour de France, je me sonviens que fen le Marquis de Croissy, alors Minisire et Secretaire d'Estat des affaires étrangeres, en fí confidence à l'Envoyé de Dennemarc, qui est encore en la dite Cour, Mr. de Meyercroon. Sur quoy je dirii que comme j'avois esté renvoý́ en Angleterre en 1678, et où je restai jusques an commencement de 1680 , que je fus Envoyé en France de le part do fen Sa Serenissimeté Electoralo (apres aroir déja esté sabsiitá par ses ord̄es an Comte, alors Baron de Schwerin, son Envojé en Angleterre) anssi durant mon dit séjour à Londres plusieurs y aroient en veue de marier la dite Princesse Anne avec Ie Prince d'Hanovre l'aigné, sujourdhuy Electeur de Branswic, ${ }^{13}$ daillears fils d'une Princesse 14 du Sang d'Angleterre, et appellée depuis a ls succession d la Couronne. Et comme on sçavoit que j'avois l'honnewr d'estre en quelque commerce de lettres avec lo äto Serenissime Princesse ss Mere, je fas requis de quelques personnes de consideration en la Cour d'Angleterre, de lay en écrire, et à ce que le Prince son fils fus envoyé en Angleterre is ce sujet, pour s'y isire connoistre. Et sur ce que je pris une fois la liberté de luy toucher quelque chose en passant, dans une de mes lettres, de ce qu'on m'en insinuoit is Londres, Ia dite Altesse, aujourdhay

- Que addea by Spanheim.

- 1702.

- Corrected by Spanheim out of ' 1685 ,' as it secms 4 Not. 1.

"Aug. 7. iz De mome ... York, marginal note by Bpanbeim.

12 George Lewis, afterwards Fing Gearge I of England. 14 Tho Eloctress Sophia. 
Electrice de Brunswic me fit connoistre par sa reponse, qu'on n'y estoit gueres aisposé $\dot{z}$ Eanovre, et entr'autres veu la naissance de la Princesse Anno da casté de sa Mere, née d'une famille tort médiocre. Anssi ce mesme Prince son tils, que j'eus l'honneur de trouvér à Paris à mon arrivee en 1600, estant passe de lis en Angieterre, sur la tin de is mosme anriè, y temoigna peu d'attention iे un mariage avec la dite Princesso; et en parrit en sorre qu'on jugeoir bien, qu'il n'auroir pas lieu; et ce que la dite Princesse Anne, à ce qui m'a esté dit plus d'une fois, et d'assez bon lieu n'auroir pas oublié. Ce qui spres tout donns occsasion d la Cour de France, de songer en suite d̀ l'étsiblissement àe cetto Princesse, et pour au besoin entraverser en Angieterre les pretensions on le parai, que le Prince d'Orange, Mari de ls Princesse aimée, pourroit y avoir, et contre lequel on estoit forr prévenn en la dite Cour. Ls Princesse Anne, dépwis son mariaye, vécut an grande concorde et union avec le Prince son Epoux. II s'y trouva mesme queique conformité d'humear, is aimer plustôt le parvicuiier et la retraite, que le granả monde, et les divertissemens d'éclat. Lors que je fus envoyé en Augleterre en 1685 a faire les complimens au Roy Jaques son Pere, sur son avénement à la Couronne, Elle avoit pour sa Dame d'honneur le Corntesse de Clarendon, Dame de merite, et de verta, et femme du frere aisné de feu is Duchesse sa Mare.

\section{De la naissance äu Duc de Glocester son Fills.}

Certe Princesse vint à estre plusieurs fois enceinte, mais sans porter ses enfans à terme, et ainsi d̀ faire des fausses conches, jusques au nombre de quatorze. Ce ne fat qu'eu mois de Juillet de l'année 1689, et ainsi apres ls revolntion, qu'Elle accouchs à Hamproncourt, d'un Prince en 1689 resté en vie, appellé du nom da Duc de Glocester et qui a vescu jusques à l'année $1700,{ }^{15}$ qu'il mourat à Windsor, âgé de onze années et quelques mois.

\section{De la Bovolution arrixces on Angleterre, et du parti qu'Elite prit avec le Prince son Epoux.}

La Reine d'Angleterre, ${ }^{16}$ Epouse da Roy Jaques, Catholique Romaine, et née Princesse de la Maison du Duc de Modene en Italie, se trouvoit aussi sans enfans, jusques à la mesme anné 1688, qu'on la publia enceinte, et estant venue à terme d'acconcher, d'estre henrensement délivré d'un Prince, appellé suivant la coustame des Heritiers de la Couronne, du nom de Prince de Craies. Je n'entrerai pas icy dans la discussion de la verité, on supposition de cette naissance; ce qui ne regarde pas le sujet, dont $\nabla=$ Sija $^{\omega} \mathrm{m}^{\prime} \mathrm{a}$ commandé de luy rendre compte. Je airai seulement par rapport à la Princesse de Danemarc, que s'estant trouveé aux Bains de Báth an remps de lo naissance veritable ou prétendue de ce Prince de Gales, Elle fat prevenue qu'il y avoit du mystere et du manege dans la naissance de ce prétendu Frere, et contribus à affermir la Princesse d'Orange sa soeur dans la mesme créance. Ein sorte que dés l'arriveé da Prince d'Orange en Angleterre en Novembre 1688, Elle prit le parti de se laisser enlerér par l'Evesque de Londres, qui avoit esté autrefois Capitaine de Cavaierie, et pen affectionné an Roy Jaques; et

" The AS. has 1670, ef peg 771.

16 Jary Beatrix Eleonora 
que le Prince de Dennemarc prit anssi celuy, ${ }^{17}$ de quittér le Roy son Beau Pere, et se rendre vers le Prince d'Orange.

La revolution d'Angleterre étant ensuite arrivée, par la retraite da Roy Jaques en France, précedé déja aupararant de celuy de son Epouse et du prétendu Prince de Gales; et le Prince d'Orange et la Princesse Marie son Epouse déclarés Roy et Reine d'Angleterre, en Fevrier 1689, la dite Princesse pessa de la Haye où Elle estoit resteé jusques lì en Angloterre. Ce qui donno lien \& l'entrevae des deax soeurs, qui ne s'estoit pas fait is depuis donze années, qui fat celle da Mariage de la Princesso d'Orange en 1677.

\section{D'uns desurion arrives ontre lo fou Roy ot Reine d'Angleterre et entre la dite Princesse.}

Le Prince et lo Princesse de Danemarc vécurent depuis dans une assez grande dépendance du nouvesn Roy et Reine jusques à nne desunion, qui arriva entre les denr Soeurs, à l'occasion de la Dame d'honneur de la Princesse. C'estoit la Comtesse, anjourd'huy Duchesse de Marlborough, dont il g ara lien de parler cy-apres. Je me contenterai de dire icy, que cotte Dame arant son Mariage avoit esté fille d'honneur de la Princesse, et pris des lors un grand ascendant sur son esprit: Qu'agant éponsé en suite Mylord Churchill, anjourd'huy Dac de Marlborough, qui estoit en grande faverr anpres du Boy Jaques, tant par son merite, que pour estre frere de la maitresse de ce Roy, ${ }^{19}$ Elle fat faite premiere Dame d'honnear de la dite Princesse. Ce qui ayant angmenté sa consideration et son crédit sur l'esprit de sa maistresse, en sorte qu'il paroissoit qu'Elle s'en laissoit entierement gouverner, donns lien an Roy et à ls Reine, qui le 80 royoient avec déplaisir, et en craignirent les suites, de desirer de la Princesse, qu'Elle congediast sa dite Dame d'honnear, et en prit nne autre \& sa place. Mais quelque instance, qui luy en fât faite de leur part, Elle ne voulut point y donner lien; et préfera de s'exposér a leur disgrace, et \& tout le ressentiment, qu'ils en axroient. Ce qui alls sussi si loin, qu'on osta les Gardes à la dite Princesse, qu'Elle se crat obligée d'abandonner son logement à la Maison Royale de S. Jemes, et de se loger avec ${ }^{21}$ le Prince son Epoux dans noe maison particuliére, et occupé aujourd'huy par le Duc Devonshire. Elle demenra sassi quelque temps dans ane maison de campagne, a quelques milles de Londres, qu'on appelle Sion, et qui appartient an Duc de Sommerset. Cette desunion dara deax années entieres, sans que les deas soeurs, la Beine, quoy que demeurant a Londres, et la Princesse se soient venes depuis, ni mesme à la maladie de ls Reine dont Elle mourat. Ce qui estoit arrivé, depuis que lo Reine estant allé visiter la Princesse sa soenr, attaqué 23 d'une assez grande maladie, en suite d'une fausse conche, et lay parlant encore d'oster la Comtesse de Marlborough d'aupres d'Elle, la Princesse lny anroit dit, que si la

17 Celary written by Spanhoim instead of la parti.

- Correctod by Spanheim inatead of s'etoint pas vesus.

- Atter Boy the MS. has at duqual il y aura lieu auseri de parlor dars la suito blotted out.

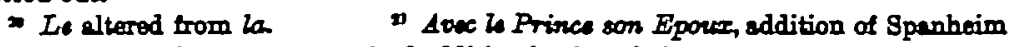

n Elle . . . Sommersat marginal addition by Spanheim.

- Altered trom dans une. 
Reine ne venoit la roir, que pour luy parler contre la dite Dame, Elle pourroit se dispensér de revenir une autre fois, et en se tournant $"$ en mesme temps, à ce qu'on sjoûte, de l'antre costé de son liet.

\section{De sa conduite depuis la mort de la Reine sa sasur et jusques a celle du fou Roy.}

Apres la mort de la Reine arrivé en 1695, le Prince et la Princesse eurent permission du feu Roy de reprendre lear logement an Palais de S. Jemes. Ils y ont véeu jusques à sa mort ${ }^{25}$ dans une grande tranquillité, sans besucoup d'eclat, avec une Cour assez mediocre, et sans aroir ni pretendre quelque part dans le gouvernment, on en avoir aucune dans la confidence du feu Roy, qui visitoit rarement ls Princesse, et ne donnoit sussi gueres lieu au Prince de l'entretenir. In est difficilo, s'il m'est permis de dire, avec tout le respect den a la mémoire du feu Roy, de donner bonne raison de cette froideur et indifférence, dont on voyoit qu'il usoit en lear endroit; quoy que la dite Princesse se belle soeur deut heriter de ${ }^{26}$ ses trois conronnes apres sa mort, que la constitution infirme du mesme Roy faisoit ${ }^{27}$ craindre que cela n'arrivast bien tost ; et ainsi ce qui auroit pú ce semble donner lien, à en témoignér plus de consideration etre dailleurs veu que ls dite Princesse et le Prince vivoient 28 dans une espece de retraite, quoy que dans une grande ville, et aveo une conduite, qui ne pouroit pas donner le moindre ombrage. Il y eat une occssion en la derniere anné de ls mort du Roy, qui pât contribuer à cette froideur. C'est qu'ayant esté adverti, que la Princesse auroit recen une lettro da Roy Jaques son Pere, sans en parler, on la produire, il l'alls tronver pour lay demander à la voir. Ce qu'Elle anroit évité, en avouant de l'avoir recen, mais de l'avoir bruslé. Le feu Roy en auroit aussi parlé, et en des termes assez forts, à la Comtesse de Marlborough, dont la soeur, Duchesse de Tirconnel, 29 (qui a eu permission sous ce Begne, de retourner en Irlande, et vient d'en faire un tour icy à Lrondres) estoit alors Dame d'honnear \& S. Germain de la Reine Epouse da Roy Jaques. Le Prince de Danemare estant venu à Kinsington pour voir le Roy dans les derniers jours de sa maladie, et qu'on craignoit, ce qui en arriva ; a peine lay donna-t-on lieu d'entrer dans la Chambre da Boy, et luy faisant connoistre qu'il feroit bien de n'y gueres rester; en sorte qu'il ne fit presque qu'y entrer, et sortir. Pour la Princesse, Elle témoigns aussi d'spoir dessein de se rendre \& Kinsington, et en tout cas qu'Elle resteroit dans l'Antichambre.

\section{De son avenement d la Couronne.}

Los mort da fen Roy, arivée ${ }^{30}$ en éffet au dit Kinsington, un Dimanche matin $\frac{8}{19}$ Marg 1702, donna lien \& voir la Princesse de Dennemarc reconnue en mesme temps Reine des trois Royrames; proclamée telle solemnellement par la ville de Londres dés le mesme jour apres midy ; et complimenté en cette qualité de la part des deux Chambres du Parlement,

* Thos corrected by Spanheim instead af trowarint.

- Spanheim has corrected la mort du fou Roy.

* De merginal note by Spanheim. $\quad \Rightarrow$ Faisoit corrected by Bpanheim.

- Vivoint corrected by Spanheim instead of vecussent.

- Oui . . I Londres and Tirconnal by Spanheim.

- Corrected by Spanheim instend of estant en dffet arrioce. 
qui s'estoient lì dessus asgemblés exnrardinairement le mesme jour ; et do Maire et deg Echevins de la viile de Irondres.

$J_{\theta} n^{\prime} a y$ pù que toucher en premier lieu et sommsirement les circonstances susdites, quoy que dailieurs assez connues qui regardent la personne de la Reine, en quaiité de Princesse dnne et en suite de Princesso de Dennemarc, avant son avénement ì is Couronne. Il s'agit maintenant de la considerer depais qu'Elle est monté sur le Throne de trois Royanmes, abancionnés par le feu Roy Jeques II son Pere. Ei à ce sujet il y aura lien de reflechir sur les considerations, que j'ay allegueés des l'enureé de cét Ecrit ; à sçaroir, de ls constitution de sa Personne, de ses quaiités, et inclinstions, et ainsi du veritable caractere de son Esprit et de son narurel; de sa conduite dans le domestique, et a l'égard dn gonvernement et des affares; des Ministres, ou daillenrs des Dames, qui ont le plus de part en 89 confidence, et ${ }^{31}$ en son amitié, et ainsi les plus accredités et autorisoés anpres d'Elle, enfin de l'estat present de sa Cour, particulierement par rapport aux Ministres étrangers qui y sont envoyés.

\section{De la constitution dis sa personne. 32}

La Roine seroit d'assez belle taille, hors qu'Elle est accompegné de trop d'embonpoint ; bien prise an reste en 98 personne; les chevear noirs, les yeax bleads, le nez, la bouche, la gorge, les bras et les mains belles. Le taint du visage est souvent brovillé, et accompagaé de quelques bontons. Son air est nairarellement serieax, mais dailleurs qu' Elle prend à tasche de rendre affable et graciear envers les personnes, qui ont l'honneur de l'aborder, ou qu'Elle honore de son entretien; et en quoy Elle conserve tous les differens égards, que le rang, ou le merite des personnes peut demander. Elle est fort propre en ses ajastemens, quoy que sans affecisioion, recherchant plus ls bienseance et le bon goust, que l'eclat et l'osientation. Anssi ne porie Elle pas d'anires joyanx sur Elle, hors des occasions extraordinaires de cérémonie, que cenx qui accompagnent le 8. George, qu'Elle porte attaché à un raban blen an hant de son corps de jupe, et comme en place d'agraphe de diamans. Sa coiffure est fort nsturelle, ei son maintien agresble. Son temperamment paroist assez robuste, hors le malhear qu'Elle a eu de faire tant de fausses couches; et que depuis quelques années, Elle se trouve sujette à des stieintes de gouie a la main et aur genoux, 23 quoy que sans grande doulear, et qui l'obligent seulement à garder la chambre. Elle y a cherché du soulagement ces deur anneés passés par l'usage des eaux chandes de Bath, qu'Elle y est allé boire vers l'Automne, et áoni Elle a cren de s'estro bien trouvé. Elle esi sobre dailleurs dans son manger et dans sa boisson; bien qu'on ait debité quelque fois dans les pays étrangers et à tort, comme si Elle avoic du penchant \& des excés a boire, et qui auroient contribué à ses fansses conches, et ì ses atteintes de goute. Enfin il y a lieu de juger par sa constitution, qu'Elle peut encore remplir une longue carriere, avant que de donner lieu à un successeur; à moins que ses acces de goute ne devinssent plus frequens, et plus fachenx, qu'ils n'ont été jusques icy.

n Et added by Spanheim. This tixle is not by Spanheim. * Altered tram pichs. 


\section{De ses qualites et incinations.}

A l'égard de ses qualités personnelles, et de ses inclinations on peut dire, qu'slle a natarellement dans l'sme de la bonté, de la donceur, de la retenue, de ls franchise, et de l'honnesteté, si on pent se servir de ce dernier mot, en parlant ${ }^{M}$ d'une grande Reine. Je ${ }^{35}$ dois ajouter qu'en premier lieu, Eile a besucoup d'attachement à la Beligion; à assister régalierement tous les jours sux exercices de devotion de l'Eglise Anglicane; et a commanier tous les premiers Dimanches de chaque mois. Qu'aingi Elle eat ennemie de tonte profanation et de libertinage en matiere de Beligion, ou autrement : qn'Elle n'est pas moins bonne Femme, que bonne Chrestienne, ayant toujours vécu, comme $\mathfrak{i l ~ a ~ d e ́ j s ~ e s t e ́ ~ r e m a r q u e ́ , ~ d a n s ~}$ une grande Union avec le Prince son Epoux; et qui continue avec lo mesme force et sur le mesme pied, que lorsqu'Elle n'estoit que Princesse, gardant tonjours pour luy les memes égards: en sorte qu'ils sont comme insèparables, et se trouvent toujours dans on mesme lieu. C'est dont Eille a encore donné des marques, depuis qu'Elle est Roine, assez éclatantes ot assez publiques, en faisant donner la oharge de Grand Admiral. la plus considerable en Angleterre, et surtout dans le temps d'une grande grarre et d'anssi grosses flotes sur pied, dailleurs in portant le Parlement a lay assigner nne anssi grosse pension, que celle de $\frac{100}{m}$ Livres Sterl. annuels en css qu'il vienne \& survivre \& la Reine. On pent juger que la conformité d'humeur et d'inclination a aimer plus le particulier et la retraite, que le grand monde, $y$ contribue. Anssi n'y a-t-il, que certains jours dans la semsine, od ls Reine a coustame de se rendre visible, et de tenir cercle; et an reste $\pi$ ne donne gueres lieu an spectacles et anx divertissemens publics de danse, de musique, on de comédie, gn'en des jours extraordinaires, et qui y sont comme destinés par ls coustame. Elle a aimé autrefois ls danse et ls musique : dansoit Elle mesme avec besucoup de justesse ; mais I a renoncé depuis qu'Elle a commencé a grossir, et avoir des atteintes de goute. Les divertissemens dailleurs se sont rendus moins fraquens à sa Cowr, et n'estant encore que Princesse de Dennemare depuis ls mort du Duc de Glocester son Fils, et qui devoit estre son successeur ì ls conronne. En sorte que ces divertissemens ordinaires icy d Londres so reduisent an jea de'la Bagsette, a quoy Ello donne lien les soiroés destiness \& tenir Cercle.

Co que je viens de dire fait an reste, que pour nne corr telle que celle d'Angleterre, et dans nne anssi grande ville, st remplie, surtout l'hyver, de personnes de qualité des dowr séxes, comme de Mylords, Pairs ${ }^{38}$ on Pairesses da Royanme, elle ne repond pas, quand on ls roit, à l'attente des étrangers, ni à l'inclinstion de ls Nation, qui voudroit ls vair ordinairement plus grosse, et où il y eut plus d'éclat et do divertirsement. $A^{29}$ quoy contribue d'ailleurs le séjour que ls Beine fait tout l'Este, et

- Carrocted by Spanhoim instead of d I sgarch.

- Je dois ajouter qu'em, marginal noto of Spanheim instend of Qu'en of the tent.

- Gaddart . . e egards merpinal noto by Spanheim.

* At reste by Spanheim instead at que darlfersos Elle.

- Pairs oes Paireseses du Royaume added by Spanheim.

- A quoy Le . . que la Reine by Spanheim, ingtead of Ce que . . a aus sejors qu' Ethe of the first tert 
partie de l'A utomne à Windsor, où ls Cour esi fort petite, hors le Hercredi avant disner, et particulierement le Dimanche, qui est le jour du Conseil du Cabinet, et ainsi que les Ministres, qui en sont, ont coustume de s'y rendre. Ce sont dailleurs les deur jours, que les Dames de qualité de Londres, on du voisinage, on autres personnes, qui ont quelque relation ou affaires en cour, prennent, pour s'y rendre et s'y faire voir. La Reine de son costé n'y prend gueres d'autre divertissement, que celuy de la promensde, et de la chssse dans ls Forest de Windsor, et dsilleurs, comme j'sy dit, celny du jeu de la Bassette.

\section{Dus caractere de son dsprit.}

On peut dejja ancunement juger, par ce que dessus. ${ }^{10}$ que le veritable caractere de l'esprit de la Reine, est plustot de l'avoir porté à la justice, à l'equité, à la donceur, anx égards et menagemens requis, od il en fant avoir, envers les Mylords et Dames de lo Nation, on des Ministres étrangers; que d'avoir un tour d'esprit brillant, qui aime à se produire, et a se faire valoir par la conversation, et des entretiens sonstenus, et de qualque durée. 41 D'où on peut recueillir, qu'Elle parle pen et avec ménagement, deilleurs arec agrément et bienséance. Qu'au reste Elle a de la docilité, pour se conformer aux advis des personnes éclairés, et en qui Elle a de la confiance ; et d'antrepart de la fermeté dans ses inclinstions, envers les personnes, qui en sont honorés, et en qui Elle a pris quelque créance particuliere: Que cela se purt déja assez reconnoistre, de ce qui a esté touché cy-dessus, estant Princesse de Danemarc, sur le sujet de la Coimtesse à present Duchesse de Marlborough se Dame d'honneur, et de laquelle 12 il y aura lien encore de perler dans ls suite.

Ce 43 caractere de l'esprit et dn natorel de la Roine paroist également dans sa conduite domestique, ausi bien que dans celle \& l'egard du gouvernement et des affaires. Comme il a déja esté remarqué qu'Elle se plaist dsrantage dans le particulier, que dans le grand monde, cela fait sussi qu'Elle ne se communique gueres familierement, qu'svec les personnes qu'Elle a d̀ son service, et pres de sa personne, et qui sont de trois sortes; ses Dames d'honneur, ses Filles d'honneur, et ses Femmes de Chambre, qui suivant la coustame d'Angleterre, sont des Filles on Femmes ( $y$ en ajant des deux sortes en cette 44 fonction) de bonne maison, et bien apparentées. Mais c'est dont il sera parlé cy-apres.

\section{De ses occupations, de son Conseil du Cabinet et de ses principaur Ministres.}

Cette mesme inclinstion de la Roine, fait qu'Elle passe lo pluspart dn tems parmi son domestique, hors ${ }^{45}$ celny qu'Elle est obligé de donner aur affaires du dedans ou du dehors de ses Royaumes, à l'entretien de l'un ou de l'sutre de ses Ministres, et aux Conseils, qui se tiennent devent Elle. C'est a quoy Elle s'occupe aussi souvent, et autant de fois, que le besoin et les affaires le requierent. $D$ est rray, et surtout darant le

- Que dessus corrected by Spanheim, instead of que jo vions de dire.

" Durce cocrected instead of durete. De Laquelle by Spanheim, instead of dont.

- Co . . . de la Reire, Spanheim, instead of C'at auri ce qui.

- En citte fonction, marginal note by Spanheim.

s Hors added by Spanheim abore the line. 
séjour qu'Elle fait à Windsor, que hors ${ }^{46}$ les cas, qui surviennent à la traverse, il ne se tient gueres de Conseil du Cabinet devant Elle, que les Dimanches au soir. II y a mesme quelque fois des Dimanches, que par le retard des ordinaires de delà la mer, ou antre raison, qu'il ne s'en tient mesme point an dit jour. Les séances de ${ }^{47}$ ce Conseil sont ordinsirement plus frequentes dans le temps de la teneur d'm Parloment; reu les incidens, qui y surviennent, et qui ne penvent qu'y donner lieu. I n'intervient dsilleurs à ce Conseil, que les Ministres, qui y sont appellés par la Roine, et qui lors qu'ils sont tous en ville, ou au voisinage, se reduisent à l'Archevesque de Cantorbery; an Grarde des Scesux; su Grand Thresorier d'Angleterre, Mylord Godalphin; an Comte de Pembrock, President da Conseil, à sçavoir du Conseil Privé, qu'on appelle, on Grand Conseil ; an Duc de Normanby. Garde des Scesux privés ; au Due de Devonshire, Grand Maistro de la Maison de la Reine; au Duc de Sommerset, Grand Escuyer ; an Duo de Marlborough, quand il est en Angleterre; an Duc d'Ormond, quand il est a Londres, comme Viceroy d'Irlande; su Comte de Rochester, qui s esté son Predecesseur en cet employ, msis qui affecte depuis plus d'un an an ca de ne s'y point trouver: et aux deux Secretaires d'Estat, aujourd'huy le Chevalier Hedges, et l'Orateur de la Chambre des Communes, Robert Harley. C'est dans ce Conseil, qu'on traite les affaires secretes, soit du dedans on dehors du Roysume ; quil se fait ts la déclaration des charges ou changemens des Officiers de la Couronne, ou de la Maison de la Reine; du choix des Ministres an dehors, de leurs Instructions, et des rapports qu'ils penvent avoir fait par leurs lettres aux deux Secretaires d'Estat, suivant leurs deux differens départemens: Et ainsi en general des Traittes; des Alliances; des Memoires on remonstrances faites de la part des Ministres étrangers en cetto Cour ; enfin, arant et durant le tomps de ls convocstion d'un Parlement, à digerér les cas et les affaires, qu'on y doit proposer, appuiér on detourner de la part de la Cour. Il arrive quelquefois, suivant les occurrences et les affaires, qu'il n'y a qu'une partio de ces Ministres susdits, et les plus affidés, qui sont appellés an dit Conseil. ${ }^{49}$ Daillears il y a bien des affaires, et surtont celles qui peurent regardér le dedans de la Cour, la disposition des charges, les ménagamens entre les deux partis des Thorye, et des Wights, les graces et les bienfaits de la Reine, dont Elle ne se rapporte qu'è ses Ministres les plus considerés et les plus accrédités, comme sont le Grand Thresorier Mylord Godolphin, et lo Dac Marlborough, lorsquil est en Angleterre.

Sur quoy et au sajet de ces deax partis, qui font assez de bruit et an dedans et au dehors de l'Angleterre, je dois remarquer qu'ils se fomenterent et avec pen de menagement l'un envers l'autre sous le Regne du fen Boy Guillanme. Que le nom de Thorys, qui s'estoit donné anx partisans les plus zéles de l'Eglise Anglicane, et pour l'affermissement de l'sutorité Boyale en Angleterre, se communique en suite a ceux d'entr'eux qu'on crút pen affectionnés d̀ la personne et d l'administration da fen Boy, qui mettoient en doute ls validite de son droit et avenement a la Couronne, et dailleurs étoient peu enclins, et à entrer dans tous les

- Que hors corrected by Spanheim, instead af hors.

"De ce Conseil corrocted by Spanhoim, instead of am

- Quil se fait added by Spanheim. - Corrected trom aur dit Consoils. 
engagemens. qui tendoient a toute exclusion du prétendu Prince de Gries. ou à g'interesser anssi avant dans les guerres do deld̀ la mer. Io parti deg Wights dautre part. estoit caluy en general qui aroit en le plus de....30

\section{Do Mrylord Godolphin Grand Thresorior d' Angleterre et lo caractere de sa personne.}

Mylord Godolphin est d'une bonne et ancienne famille d'Angleterre, quoy que le premier Mylord de ce nom. I se fit déja connoistre et dis. tinguer par son merite sons le regne de Charles II; et depuis sons celny du Roy Jaques, sous lequel il fat fait Mylord Baron et Pair da Royaume. In fut employé sous son Regne dans l'administration des Finances, an qualité de Commissaire de l'Echiquier, comme on les appelle an Angleterre, et par la grande et particuliere habileté, qu'il I fit paroistre. fust continué dans cette mesme fonction sons le feu Boy Grillsume. et mesme étabii le Chef. ou le premier des Commissaires de l'Echiquier. Ce qui a lien, lors qu'il n'y a point de Grand Thresorier d'Angleterre, comme il n'y en a point eu durant tont le regne du dit Boy, et qu'en ce cas lì. l'administration des finsnces est mise en commission de quelques personnes, $d$ qui on en donne lo soin, et parmi les quels il $\mathrm{y}$ en a un, qui en est établi le Chef, et ainsi en cette qualité a ${ }^{51}$ la principale direction, mais au reste sans avoir le rang, les apointemens, ${ }^{52} \mathrm{ni}$ la consideration de Mylord Thresorier, ni aussi en portér le nom. Ce ne fut que deax ou trois mois avent la mort du feu Roy, qu'ayant resolu de casser le Parlement, qui devoit se rassembler dans ce mesme mois de Decombre, et composé la plus grande partie du parti des Thorys, le dit Mylord Godolphin, pour ne pas devenir suspect su mesme parti. et dont il a toujours esté, vint resigerer an fen Roy se commission de Chef de l'Eahiquier, et sans la vouloir garder, quelque instance que le Roy luy en fit.

La Reine pen de tomps apres son avenement a la Couronne le déclara Grand Thresorier d'Angleterre, qui est consideré pour la plus grande charge de la Cour et du Roysume, et luy donne anssi le premier rang, spres l'Archevesque de Cantorbery, et le Chancelier on Garde des Sceanx, ef tire ordinsirement apres soy ls consideration de Premier Ministre, bien que sans en porter le titre. II n'y ent que le Comte de Bochester, frere comme il a esté dit de fou la Duchesse d'Yorck mere de la Reine, qui avoit déja exercé cetto charge dans les premieres annés du Regne du fen Roy Jaques II, et qui s'attendoit de rentrer dans cetto importante charge, lequel en conçeut wo déplaisir sensible, et s'est abstent depuis de prendre part aux affaires. Co choix eut dailleurs l'approbation generale et mesme du parti des Wights, aussi bien que des Thorgs; ver la grande habileté connue du dit Mylord, joint a son grand desinteressement, pour l'exercice de la dite charge, et la repatation établie, que personne n'en estait plus ospable que luy. Ce qui a ansai esté confirmé jusques icy, par lo bon estat, où il a mis les finances de ls Reino; trouvé moyen do fournir sux depenses extraordinaires, ì quoy les conjonctures publiques durant ls presente guerre, surtout l'alliance aveo le Portugal et ses guites, les assigtances d'argent à l'Empereur, aux deux Cercles de Franconie et du Susbe,

$$
\text { - Sia "Ms.L. Corrooted trom apartemense. }
$$


et $\$ 3$ antres ont donné lieu, et avoir encore des fonds de reste, su bout de l'année. En sorte que le caractere du dit Mylord Grodolphin est d'aroir une grande ézactitade et application, pour l'exercice de cette importante charge: d'evitér a ce sujet ce qui pourroit l'en distraire; et aingi à ne paroistre pas d'entrer dans le détail des autres affaires de la Cour et du gonvernement, quelque bonne part dailleurs qu'il $>$ ait par son credit, et par sa confidence et liaison avec le Duc et la Duchesse de Marlborongh, et par là ne donner gueres lieu à estre visité des Ministres

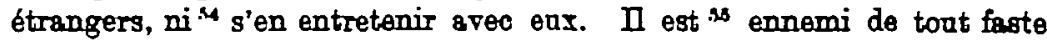
et de toute parade extèrieure en son domestique, en son train, et en tonte sa conduite, et pent estre jusques à l'excés, dans le haut poste où il se troure; et bien que le premier en so rang par sa charge, entre les Pairs secoliers du Royaume, se contentant jusques icy de la derniere qualité des Mylords, qui est celle de Baron, et ayant consenti avec peine a estre fait, comme il vient l'estre depuis quelques semaines, Chevaliar dela Jarretiere. $I$ parle pen et aveo beancoup de retenne. Apres tout il ne laisse pas de conservér une passion, qu'il a toujours eue, pour le jeu; et ainsi d'y donnér les henres d'apres disner on da soir, qu'il en pent trouvér le loisir. I aime anssi besncoup le divertissement des courses de chevanx, qui se font en Angleterre avec plus d'attachement et de depense qu'ailleurs, et ne manque jamais de se tronver à celles, qui se font denx fois l'année en Printemps et en Antomne, ì Neumarcket. $I^{37}$ est lié particalierement d'wne ancienne et étroite amitié, comme ${ }^{\text {so }}$ il a deja esté remarqué, avec le Duc et la Duchesse de Marlborough depuis longues anness, et qui s'est angmenté par le mariage de son Fils aigné, arec lo Fille aisneé de ce Duc. En sorte que le Duc de Marlborough se trouvant sans fils masle, depuis la mort de celay qu'il aroit, il y anrs un an et demi passé, on croit que son titre et dignité de Dac, pourra passer apres sa mort à son Gendre, fils aismé du Mylord Godolphin. C'est dailleurs cette mesme lisison, qui peut estro n'a pas pen contribué à mettre ce Mylord dans la confidence particuliere de la Reine, et à la charge de Grand Thresorier d'Angleterre plustot que le Comte de Rochester, son oncle maternel, et qui y aspiroit ; comme il a esté dit cy-dessus. An reste ce Mylord est du nombre des Thorys moderés, fort porté dejllenrs is lo poursuite de la guerre presente. 87eo vigueur; ${ }^{39}$ an maintien des Alliances faites à ce sujet, et \& lo sonttenir.

\section{Du Duc de Marlboroug, du degres de son elevation at du caractere de sa personire.}

Quant an Duc de Marlborough, il a déja l'honneur d'estre conna personnellement de $\nabla^{=} \mathrm{Maj}^{\mu}$, pour me pouroir dispenser d'en faire icy le portrait ; outre que les grandes et glorieuses actions de cette campagne, qu'il vient de faire vers le Danube, contribuent is en faire les plas beaux

\footnotetext{
w Et . . . lariwe added in margin by Spanheim.

4 Ni corrected by Spanheim.

4 Il cat mitten bJ Spanheim instead of Aussi est ith

- En added by Spanheim.

" Corrected by spanhoim from Au reste if.

- Comms . . . romarqu added by Spanheim.

- Lvec viguerr marginal note by Spenheim.
} 
traits. Je dirai senlement, que ss famille est Churchill; qu'il est fils d'un Pere de ce nom, qui estoit Chevalier Baronnet; et qui fut Envoyé en Dennemarc sons les regnes passés: Que son Grand Pere du costé maternel estoit an des freres cadets du premiér Dac de Buckingham, farori du Roy Jaques I, et par où lo dit Mylord se trouve proche allié de tous ceur de la famille des Villers, dont estoit ce Dac. Mylord Pembrock President du Conseil m'a dit, que sa Grand mere du costé maternel estoit soeur du Grand Pere du Duc Marlborough et ainsi soeur pareillement du Duc susdit de Buckingham, elevés à cette dignité par la favear da Roy Jaques L D'où il resulté, que le Duc de Marlborough, n'est pas veritablement d'une extraction de famille de Mylord d'Angleterre, mais apres tout n'est pas d'une naissance aussi obscure, que ses envieux on ses ennemis le veulent faire croire par deca. ${ }^{61}$ II naquit veritablement sans beaucoup de biens de fortune, et à ce qu'on prétend, fut redevable de la premiere qu'il eut, à l'inclination pour luy de la Duchesse de Cleveland, maistresse du feu Boy Charles II, et dont il auroit en une gratification de $\frac{10}{m}$ Livres

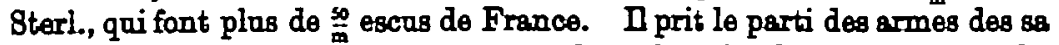
premiere jennesse, et fut Lieutensant dans les Gardes du Roy susdit Charles. Sa soeur Churchill estant devenue ensuite ls Maistresse du Duc d'Yorck depuis Roy Jacques II, et dont il eut deux fils le Duo de Berwich, qui commande anjourd'huy en Espagne, et le Duc d'Albemarle, comme on l'appelloit à S. Germain et à la Cour de France, décédé su commencement de cotte gaerre en 1701 et ane fille presentem[ent] a Londres, cels contribua à avancer le dit Duc de Mariborough son frere. En sorte qu'il fut fait Mylord Baron d'Angleterre, en suite de l'avenement du Duc de Yorak à la Couronne sous le nom de Jaques II, en fat envoyé en France, y faire des complimens, durant mon séjour precedent on ls dite Cour, en 1685, et se trouvoit fort avant dans les bonnes graces de ce Roy. Ce qui n'empéchs pas que vers lo tamps de ls revolution, et en suite de l'snivé́ du Prince d'Orange en Angleterre en Novembre 1688, ce Mylord vojant, que le Roy Jaques s'etolt opinistré à prendre un méchant parti, contre la Religion et l'interest de la Nation, et qu'il alloit estre abandonné de son armeé, ne prit ausai celuy de le quitter et de passer du costé du Prince d'Orange, qui dans les pramieres créations qu'il fit dès qu'il fut délaré Roy d'Angleterre, donna la qualité de Comte an dit Mylord, qui n'avait en jusques là, que celle de Baron d'Angleterre, et dsillears celle d'un des Gentilshommes de la Chambre. Aussi servit il dans les premieres campagnes 68 qu'il se firent ${ }^{63}$ an Pais bes, en suite de la dite revolution, et $\mathrm{g}$ fut dés lors fort estimé par le Prince de Waldeck, ${ }^{64}$ particolierement pour son bon sens et habileté, qu'il fit paroistre dans les Conseils de guerre. Estant de retour en Angleterre, il se fit rapport an fon Roy, de quelques discours desavantagenx do sa personne et de son gouvermement, que ce Mylord auroit tenu, et qui le touchérent si fort, qu'il luy osta sa charge de Gentilhomme de ls Chambre, et l'envoya à ls Tour. Lo Roy mesme doit avoir dit dans ce temps là que s'il n'estoit que Gentilhomme, il faudroit qu'il se vit l'epé \& la

- Elow . . Jaquas I marginal noto by Spanheim.

- Par deca written by Spanheim for icy an Argleterre

- The M8. has campagne.

it Georgo Frederick, connt of Waldeck.

- Firent corrected by Spanheim out of fit. 
main, arec ce Mylord. П sortit de la Tour quelque temps apres, mais sans rentrer dans sa charge aupres da Roy, ni dans sa faveur. Ce qui fit que ce Mylord ne continua pas de servir dans les campagnes suivantes, et qu'il resta en Angleterre, où dailleurs luy et la Duchesse sa femme, Dame d'honneur de la Princesse de-Danemarc, anjourdhuy Roine, tenoient supres d'Elle le premier rang de crédit et de fareur. Le Duc de Glocester fils unique de la dite Princesse, et son successear apres Elle à la Couronne, ayant esté tiré des mains des femmes, Mylord Marlborough luy fut donné pour Gouverneur, du vivant $d u$ feu Roy; mais ce qui ne fut pas de longue durée, par la mort de ce jemen Prince sarvenue, comme il a esté dit cy-dessus, ${ }^{s s}$ en 1700. En 1701 la grerre ajant esté resolue contre la France et l'Espagne, et à ce sujet, à conclurre les Alliances qui estoient sur le tapis, avec les Etats Generaux, l'Empereur et autres Puissances: Mylord Marlborough fut nommé du choix et consentement da fer Boy, pour commander les troupes destinbes à passer de delغ̀ la mer, au secours des Etats, et en mesme tems pour l'Ambassadear et Plenipotontiaire d'Angleterre anx dits Etats, et pour les Traittés qu'il écherroit de faire conjointement avec eax, au sujet de la guerre presente. Comme ce choir se fit durant la teneur du Parlement en Esté l'annó 1701, que je me trouvois en Angleterre Envoyé au fen Boy, je pois anssi remarquer, que le parti des Wights n'en fut gueres satisfait; et ce dans la prevention, que ce Mylord estoit on des zélès Thorys, ainsi pen affectionné au parti de la guerre, et dailleurs n'en auroit pas continué le mestier depuis plusienrs années en ça. Las suite a assez fait voir que leurs prëjugés ou craintes is dessus étoient assez mal fondées, et a justifié amplement le choix qu'en fit le feu Roy. Aussi penton juger, que deux motifs concourarent à l'y porter: l'on son juste discernement, qui luy aroit fait remarquer, et dailleurs sans ancune prérention en sa favear, comme on pent jugér par ce que dessus, les bonnes qualités de ce Mylord du costé de l'esprit, de la condnite, du génie pour ls guerre, et pour le menagement des affaires, et ainsi pour n'en voir pas de plus propre parmi les Mylords Anglois, à remplir dignement ces deux postes. L'autre motif, que cela contribuoit a la confiance du parti des Thorys, et ì les engager dautant plus dans les mesures à prendre, an sujet de la grande gaerre, oì on alloit entrer. A quoy se ponvoit joindre encore la dere consideration, ver la santé infirme du feu Roy, et qui ne pouvoit pas loy promettre une longue vie, ni ainsi autant que cette mesme guerre pouvoit durer, que ${ }^{66}$ par le choix de ce Mylord, pour remplir également ces deux fonctions de General des troupes d'Angleterre, et de Ministre pour la négotiation des Alliances, et veu tout l'honneur et les grands avantages, qui lay en revenoient, on engageoit indirectement ls Princesse de Danemarc, qui devoit succeder au fen Roy, an soustien apres sa mort des mêmes interêts et engagemens dans la guerro presento; et co veu co qui estoit connu et public du grand credit et pouvoir de ce Mylord et de la Comtesse sa femme sur l'esprit de la dite Princesse. L'evenement a aussi amplement justifié tout ce que je riens d'en dire, et au dels mesme de ce qu'on en pouvoit attendre. Vro Majo en est suffisamment instruite, aussi bien que des qualités personnelles et da caractère 
d'esprit de ce Mylord, qui pour n'avoir pas daillours esté cultivé par do grands avantages du costé de sa naissance, on de l'education, ne se trouve pas moins judicieax, solide, adroit, ferme, et affable en mesme tems; soûtend dailleurs par un dehors avantageux et une belle presence, comme on parle. J'ay pú remarquer dans le cours de la negotiation arec lay des Traittés d'Alliance des $\frac{s}{m}$ hommes ${ }^{67}$ de $\nabla$. $M^{*}$, que pour une personne, qui n'avoit pas daillears esté nourrie dans les affaires publiques, et ne commençoit que d'y entrer, qu'il aroit wn discernement fort juste, une maniere insinuante, qui alloit i son bat, sans presque paroistre de le faire, et en soutenant son advis avec beancoup de ménagement et de circonspection. Il a an reste l'inclinstion porté naturellement a l'épargne, plustot qu'à la depense; la quelle inclinstion, gi l'on en croit particulieroment ses envieux iroit à l'excés, le rendroit trop interessé, et lo senl défant que l'on tronveroit à lay reprocher. Il a fait woe perte irreparable, dans une grande elevation et haute fortane, où il se tronve, d'avoir perdu, comme il a esté dit cy-dessus, un fils wnique tres agréable et bien fait de 83 personne, de grande esperance, et qui devoit heritér apres lny de 88 nouvelle qualite de Duc, et des grands biens, qu'il n'enroit pa que lay laisser. Il a dailleurs quatre filles en vio toutes belles, trois de mariées; l'aismée, comme il a esté dit, au fils aisné de Mylord Godolphin; l'autre, su Comte de Sunderisnd; ls troisieme an Comte Bridgewater, qui a esté à Berlin, il y a deux ans passés; la quatrieme est promiso a Mylord Montalmar, fils unique du Comte de Montaiga, un des plus riches Seigneurs d'Angleterre. Je n'ay pas besoin d'ajoutér, que ce Duc est anssi entierement porté pour soustenir ls guerre presente, et les Alliances qui s'y rapportent; spres toutes les grandes et celstantes preuves, qu'il vient encore d'en donner.

\section{De la Duchesse de Marlborough at de son credit.}

Ls Duchesse de Marlborough sa Femme, est d'une extraction assex mediocre ; fut mise cependant pour Fille d'honneur anpres de la Princesse Anne, depuis Princesse de Danemaro, et anjourd'hny Roine; et s'insinus si bien dans son esprit, qu'elle en devint bien tost ls fsrorite, et ensuite sa Dama d'honneur, sous le Regne do Roy Jaques, apres son Mariage avec Mylord Churchill, aujourdhny Duc de Marlborough. Co qui angments dans ls suite, et g'effermit en sorte, que tout le crédit da feu Roy et de lo Reịne, soeur de lo Princesse, ne pust pas l'obligér à éloignér sa dite Dame d'honneur; et qu'Elle sims mienx, comme il a esté dit cy. dessus, ${ }^{70}$ essuyer tonte lear disgrace durant demr anneés de suite, que d'y donnér lien. C'est dans le mesme poste de credit et de consideration supres de la Reine, où cette Dame se trouve encore anjourdhry, et dans la fonction de se premiere Dame d'honneur, dailleurs qui garde ls bourse privé. Fille passe daillenrs dans l'esprit de la Nation, ponr avoir la premiere et plus grande part dans la distribution des graces, bientaits, et charges, dont ls Boine dispose; et pour n'y négligér pas ses interéts particuliers. Ce qui tout ensemble ne pent que lay attirér l'envie, et luy

- Treaty between Englend, Pruseis, and the General States, dated Iondon 1702, 9-19 Jan, The Hague 1701, 30 Dec.

- Corrected trom bien faitue - Comte . . troiribme an added by Spanheim.

T. Pag. 776. 
imputér mesme beancoup de choses à ce sujet, qui peavent estre mal fondeés. En sorte qu'on prétendoit mesme, que ceite grande faveur et pouvoir du Duc et de ls Duchesse aupres de la Reine, suivant l'esprit et l'hnmeur de la Nation, ne manqueroit pas de donner lien a les attaquer dsans un prochsin Parlement, ainsi qu'on s ven qu'il s'est roujours prattiqué en pareil cas, sous les Regnes passés. Mais apres tont il y a lieu de croire, que ces grandes et glorieuses victoires vers le Danube, et la derniere entr'auires vers Hochstet, ${ }^{71}$ remportées par la conduite et ls valeur du Duc de Marlborough, et qui font antant d'honneur au Regne de ls Reine, et à ls Nation, convertiront de pareilles intentions en des remerciemens et en des éloges du mesme Duc; fermeront la bouche à ses enviear et a ses ennemis; et contribueront à affermir son orédit et sa considaration dans l'esprit de la Reine, et de la Nation.

On pent juger de ce que dessus de la disposition presente de la Cour de ls Beine, par rapport d̀ Sa Royale Personne; à celles en qui Elle a le plus de confiance ; et aingi à ce qui regarde sa conduite particuliére; celle à l'egard du gouvernement et de ls Nation, ou ses Alliances an dehors. Il est constant qu'en touteg rencontres la dite Beino tómoigne besucoup de consideration poor Vostre Maj" ; it en use d'nne maniere fort obligeante envers son Ambessedeur en'cette Cour, et les personnes qui lay appartiennent, lors qu'elles ont l'honneur de lay faire lour Cour.

An reste les Ministres étrangers pour le cours ordinaire des négotiations, qu'ils peuvent svoir a traiter par deça, pour les affaires publiques et interéts de lours Principaux, ont à s'adresser au Secretaire d'Estat de leur département. 